\title{
A New Method for Solving the Initial Value Problem with Application to Multiple Black Holes
}

\author{
by John Baker and Raymond Puzio
}

1.Introduction The approaching date for broad band gravity wave detectors to come online makes the study of astrophysical sources of gravitational radiation immediately pressing. Collisions among black holes and neutron stars are especially likely to generate detectable gravity wave signals. The binary black hole collision problem which suggests itself as the natural "two-body problem of general relativity" is particularly attractive to a theorist since it allows us to restrict consideration to vacuum spacetimes. Much progress has been made toward solving this problem numerically in recent years, but the goal of a complete calculation, encompassing the inspiral of the holes, merger and the final ringdown of the remaining black hole still eludes us. Numerical techniques have been successfully applied to calculating the final stages of the collision, but there are strong limitations on the evolution time of the numerical codes as well as on the numerical domain size that these calculations can support. ${ }^{1,2}$ This means that these calculations must begin from a configuration in which the black holes are already close.

To apply general relativity to astrophysical phenomenology it is natural invoke the $3+1$ formulation. Then phenomenological problems divide neatly into two steps. We must first define initial data on a spacelike Cauchy slice which satisfies Einstein's equations on that slice and which is appropriate for the physical problem under consideration. Then we must use the remainder on Einstein's equations to integrate that data through time. Once the initial data have been specified the procedure to calculate the evolution is well defined in principle even if it may be quite difficult in practice. Thus, in principle, selection of the initial data specifies the entire spacetime. For problems of astrophysical interest it is then paramount to begin with initial data which correspond to a realistic astrophysical configuration. If the black holes begin very far apart we could approximate each as a stationary spherically symmetric black hole and there would be no ambiguity in the initial data. But once the black holes are close enough to interact significantly it is difficult to say what are astrophysically realistic initial data. Most of the numerical calculations for binary black hole collisions that have been attempted thus far have begun evolving from initial data constructed via the conformal flatness formalism. A special case of this, the so called Bowen-York method ${ }^{3}$ specifies how to derive a family of solutions to the initial value equations which have a conformally flat spatial metric and vanishing mean curvature and which can be interpreted as boosted or spinning black holes. There are problems with the assumption of conformal flatness though, particularly for spinning black holes. For an initial data set to be astrophysically realistic, a minimum requirement is that sufficiently separated black holes look like Kerr black holes. Since we do not know of a foliation of the Kerr spacetime which admits conformally flat spacelike slices, we are unable to exactly represent Kerr black holes in such a formalism. This difference between Kerr black holes and Bowen-York spinning black holes is demonstrated by the nonzero gravitational radiation emitted by a single black hole. ${ }^{4}$

In this paper we describe a novel method for solving the initial value equations of general relativity for a broad class of 3-metrics which includes axisymmetric slices. Unlike the BowenYork approach, where the extrinsic curvature is selected first and then the metric is calculated from the constraints, we first choose the metric and then calculate the extrinsic curvature. After introducing our technique, we apply it to the Kerr black hole demonstrating that the Kerr hole does indeed come out as a special case. By comparison with this solution, we prescribe a method which may be used to introduce multiple Kerr-like black holes on the 
initial hypersurface. In particular, if the black holes are sufficiently separated, then in the neighborhood of each one, the data approach those for a Kerr hole. Moreover, if the two holes are sufficiently close, the data also resemble that for a single black hole.

2.Solution of the Constraints In this section, we shall find the general solution to the constraint equations for a Cauchy slice whose intrinsic geometry admits a surface-orthogonal Killing field. In particular, this is true of the surfaces of constant Boyer-Lindquist time in the Kerr spacetime. Later, we will construct initial data with spatial geometry satisfying this same condition for two black holes.

For definiteness we will assume that our Killing vector field corresponds to an axial symmetry, though this restriction on the topology of the is not essential to our construction. Let $\phi$ be the azimuthal coordinate so that $\partial / \partial \phi$ is the Killing field. The existence of orthogonal surfaces implies that our spatial metric can be cast in the form $q_{i j} d x^{i} d x^{j}=\tilde{h}_{i j} d x^{i} d x^{j}+B d \phi^{2}$, where the two-metric $\tilde{h}_{i j}$ is restricted to surfaces of constant $\phi$. Since these surfaces have the topology of half-planes, we can write $\tilde{h}_{i j}=A h_{i j}$ where $h_{i j}$ is a flat metric and $A$ is a suitable conformal factor. If we choose two of the three spatial coordinates to be constant along the orbits of the isometry, then the components of $h_{i j}$ in our coordinate system as well as the functions $A$ and $B$ will not depend on $\phi$. If we choose rectangular coordinates in the half-plane, then our metric assumes the form

$$
q_{i j} d x^{i} d x^{j}=A(x, y)\left(d x^{2}+d y^{2}\right)+B(x, y) d \phi^{2} \quad .
$$

The initial value equations of geometrodynamics are a set of four coupled partial differential equations which relate the metric $q_{i j}$ and extrinsic curvature $K_{i j}$ of a hypersurface embedded in a vacuum spacetime. In tensor notation they are written as a vector equation known as the momentum constraint and a scalar equation known as the Hamiltonian constraint:

$$
\begin{aligned}
\nabla^{j} K_{i j} & =\partial_{i}\left(q^{j k} K_{j k}\right) \\
\left(q^{a c} q^{b d}-q^{a b} q^{c d}\right) K_{a b} K_{c d} & ={ }^{(3)} \mathcal{R}[q]
\end{aligned}
$$

From here we will proceed as follows. First we will show that the momentum constraint reduces to a set of Cauchy-Riemann equations to be solved for $K_{x x}, K_{y y}$, and $K_{x y}$ and the condition that $K_{x \phi}$ and $K_{y \phi}$ can be derived from a potential. Then the Hamiltonian constraint provides a condition which determines this potential. In the coordinate system introduced last paragraph, the momentum constraint looks as follows:

$$
\left\{\begin{array}{l}
\frac{\partial\left(B^{\frac{1}{2}} K_{x x}\right)}{\partial x}+\frac{\partial\left(B^{\frac{1}{2}} K_{x y}\right)}{\partial y}=A \frac{\partial\left(B^{\frac{1}{2}} K\right)}{\partial x}-\frac{1}{2} \frac{\partial(\log (B / A))}{\partial x} B^{\frac{1}{2}}\left(K_{x x}+K_{y y}\right) \\
\frac{\partial\left(B^{\frac{1}{2}} K_{x y}\right)}{\partial x}+\frac{\partial\left(B^{\frac{1}{2}} K_{y y}\right)}{\partial y}=A \frac{\partial\left(B^{\frac{1}{2}} K\right)}{\partial y}-\frac{1}{2} \frac{\partial(\log (B / A))}{\partial y} B^{\frac{1}{2}}\left(K_{x x}+K_{y y}\right) \\
\frac{\partial\left(B^{\frac{1}{2}} K_{x \phi}\right)}{\partial x}+\frac{\partial\left(B^{\frac{1}{2}} K_{y \phi}\right)}{\partial y}=0
\end{array}\right.
$$

The general solution for the third equation can be written at once in terms of a potential function $u(x, y)$ :

$$
\left\{\begin{array}{l}
K_{x \phi}=+B^{-\frac{1}{2}} \frac{\partial u}{\partial y} \\
K_{y \phi}=-B^{-\frac{1}{2}} \frac{\partial u}{\partial x}
\end{array}\right.
$$

The other two equations involve the remaining four components of the extrinsic curvature. Since there are two more quantities than equations, we may take two of the quantities as 
known and solve for the remaining two. It proves advantageous to take the quantities $P$ and $Q$ defined as $P:=B^{\frac{1}{2}}\left(K_{x x}-K_{y y}\right) / 2$ and $Q:=B^{\frac{1}{2}} K_{x y}$ as our unknowns since our equations then take the following form:

$$
\left\{\begin{array}{l}
\frac{\partial P}{\partial x}+\frac{\partial Q}{\partial y}=j(x, y) \\
\frac{\partial Q}{\partial x}-\frac{\partial P}{\partial y}=k(x, y)
\end{array}\right.
$$

where the sources $j$ and $k$ are defined as

$$
\begin{aligned}
j & :=A \frac{\partial\left(B^{\frac{1}{2}} K\right)}{\partial x}-\frac{1}{2} \frac{\partial(\log (B / A))}{\partial x} B^{\frac{1}{2}}\left(K_{x x}+K_{y y}\right)-\frac{1}{2} \frac{\partial\left(B^{\frac{1}{2}} K_{x x}+B^{\frac{1}{2}} K_{y y}\right)}{\partial x} \\
k & :=A \frac{\partial\left(B^{\frac{1}{2}} K\right)}{\partial y}-\frac{1}{2} \frac{\partial(\log (B / A))}{\partial y} B^{\frac{1}{2}}\left(K_{x x}+K_{y y}\right)+\frac{1}{2} \frac{\partial\left(B^{\frac{1}{2}} K_{x x}+B^{\frac{1}{2}} K_{y y}\right)}{\partial y} \\
K & :=A\left(K_{x x}+K_{y y}\right)+B K_{\phi \phi}
\end{aligned}
$$

These equations have the form of Cauchy-Riemann equations with source and can hence be solved by well-known methods of potential theory. For instance, we might write $P$ and $Q$ as integrals of $j$ and $k$ against Green's functions.

$$
\begin{aligned}
P(x, y) & =\oint_{\partial \mathbf{D}} d s \quad\left\{G_{\mathbf{R}}\left(x, y, x^{\prime}, y^{\prime}\right) n\left(x^{\prime}, y^{\prime}\right)-G_{\mathbf{I}}\left(x, y, x^{\prime}, y^{\prime}\right) m\left(x^{\prime}, y^{\prime}\right)\right\} \\
& -\int_{\mathbf{D}} d x^{\prime} d y^{\prime} \quad\left\{G_{\mathbf{R}}\left(x, y, x^{\prime}, y^{\prime}\right) k\left(x^{\prime}, y^{\prime}\right)-G_{\mathbf{I}}\left(x, y, x^{\prime}, y^{\prime}\right) j\left(x^{\prime}, y^{\prime}\right)\right\} \\
Q(x, y) & =\oint_{\partial \mathbf{D}} d s \quad\left\{G_{\mathbf{R}}\left(x, y, x^{\prime}, y^{\prime}\right) m\left(x^{\prime}, y^{\prime}\right)+G_{\mathbf{I}}\left(x, y, x^{\prime}, y^{\prime}\right) n\left(x^{\prime}, y^{\prime}\right)\right\} \\
& -\int_{\mathbf{D}} d x^{\prime} d y^{\prime} \quad\left\{G_{\mathbf{R}}\left(x, y, x^{\prime}, y^{\prime}\right) j\left(x^{\prime}, y^{\prime}\right)-G_{\mathbf{I}}\left(x, y, x^{\prime}, y^{\prime}\right) k\left(x^{\prime}, y^{\prime}\right)\right\} \\
K_{x y} & =B^{-\frac{1}{2}} Q \\
K_{x x} & =\frac{K-B K_{\phi \phi}}{2 A}+B^{-\frac{1}{2}} P \\
K_{y y} & =\frac{K-B K_{\phi \phi}}{2 A}-B^{-\frac{1}{2}} P
\end{aligned}
$$

The choice of Green function will depend on the domain $\mathbf{D}$ over which $x$ and $y$ range and the conditions imposed on its boundary. In addition to this solution of the inhomogeneous equations, we might also require a solution of the homogeneous system. For applications related to the two-body problem, it is natural to consider the half-plane $y>0$ as our domain and ask that the extrinsic curvature go to zero as $y \rightarrow \infty$. With these specifications, the relevant choice of Green function is given as

$$
\begin{aligned}
G_{\mathbf{R}}\left(x, y, x^{\prime}, y^{\prime}\right) & =\frac{y^{\prime}\left[\left(x-x^{\prime}\right)^{2}+y^{2}-x^{2}\right]}{\pi\left[\left(x-x^{\prime}\right)^{2}+\left(y-y^{\prime}\right)^{2}\right]\left[\left(x-x^{\prime}\right)^{2}+\left(y+y^{\prime}\right)^{2}\right]} \\
G_{\mathbf{I}}\left(x, y, x^{\prime}, y^{\prime}\right) & =\frac{2 y y^{\prime}\left(x-x^{\prime}\right)}{\pi\left[\left(x-x^{\prime}\right)^{2}+\left(y-y^{\prime}\right)^{2}\right]\left[\left(x-x^{\prime}\right)^{2}+\left(y+y^{\prime}\right)^{2}\right]}
\end{aligned}
$$

Before proceeding further with our calculations, let us pause to consider our ultimate goal. We began by specifying a metric by a choice of $A$ and $B$. Next, we chose $K$ and $K_{x x}+K_{y y}$. The solution of the equations above for $P$ and $Q$ determine two more components of the 
extrinsic curvature. The only thing we still need for a complete specification of the initial data is the $u$ potential. We shall now proceed to determine $u$ by solving the remaining initial value equation, which is the Hamiltonian constraint.

The Hamiltonian constraint can be regarded as an equation for the hitherto arbitrary function $u(x, y)$. Substituting formula 2 into the Hamiltonian constraint and rearranging, we arrive at the formula

$$
\begin{gathered}
\left(\frac{\partial u}{\partial x}\right)^{2}+\left(\frac{\partial u}{\partial y}\right)^{2}=2 v(x, y) \\
2 v(x, y):=A B^{2}{ }^{(3)} \mathcal{R}-\frac{2 B^{2}}{A}\left[\left(K_{x x}\right)^{2}+K_{x x} K_{y y}+\left(K_{y y}\right)^{2}+\left(K_{x y}\right)^{2}\right] \\
{ }^{(3)} \mathcal{R}=-\frac{1}{2 A B^{\frac{1}{2}}}\left\{\frac{\partial^{2} B^{\frac{1}{2}}}{\partial x^{2}}+\frac{\partial^{2} B^{\frac{1}{2}}}{\partial y^{2}}\right\}-\frac{1}{4 A}\left\{\frac{\partial^{2}(\log A)}{\partial x^{2}}+\frac{\partial^{2}(\log A)}{\partial y^{2}}\right\}
\end{gathered}
$$

Equation 5 has the form of a Hamilton-Jacobi equation for a particle moving in a twodimensional potential. As is well known from analytical dynamics, such a partial differential equation can be solved using the method of characteristics. Given a solution $u(x, y)$ to the equation, the characteristics are a congruence of curves with the property that the tangent vector at any point on one of these curves equals the gradient of $u(x, y)$. If we label the various curves in the congruence by a parameter $s$ and describe them by the equations

$$
\left\{\begin{array}{l}
x=X(t ; s) \\
y=Y(t ; s)
\end{array},\right.
$$

then this property is expressed analytically as

$$
\begin{aligned}
& \frac{\partial u}{\partial x}=\frac{d X}{d t} \\
& \frac{\partial u}{\partial y}=\frac{d Y}{d t}
\end{aligned}
$$

From these equations above and the non-linear partial differential equation 5 , it follows that

$$
\begin{aligned}
& \frac{d^{2} X}{d t^{2}}=\frac{\partial v}{\partial x} \\
& \frac{d^{2} Y}{d t^{2}}=\frac{\partial v}{\partial y}
\end{aligned}
$$

To solve the partial differential equation, we can reverse the logic of the last paragraph. Start with a one parameter family of solutions to the second order ordinary differential equations 6 . (These solutions can be thought of as the trajectories of a fluid of particles moving in a potential well $-v(x, y)$.) Differentiating with respect to $t$, obtain a vector field $(d X / d t, d Y / d t)$. This vector field will have vanishing curl and, by integrating, one can obtain the scalar $u(x, y)$ of which it is the gradient. For our purposes, however, only the gradient of $u$ and not $u$ itself is required, so there is no reason to actually carry out the integration. Recalling equation 2 , we can write the following formula for two components of the extrinsic curvature tensor:

$$
\begin{aligned}
& K_{x z}=+B^{-\frac{1}{2}} \frac{d Y}{d t} \\
& K_{y z}=-B^{-\frac{1}{2}} \frac{d X}{d t}
\end{aligned}
$$


Along with formulas 3 and 4 , the above formula can be used to compute the extrinsic curvature given the four freely specifiable functions $A(x, y), B(x, y), K(x, y)$, and $K_{\phi \phi}(x, y)$ and having obtained a one-parameter family of solutions to the system 6 .

3.Single black Hole In the last section, we described a method for solving for the extrinsic curvature that involved a Hamilton-Jacobi equation and mentioned that the method is applicable to Kerr spacetimes. In this section, we will consider the geometry of a hypersurface in this spacetime from the standpoint of our method. In the next section, we will utilize this representation of the initial value problem for a Kerr spacetime to construct initial data for the two black hole problem.

A stationary and axisymmetric spacetime admits a preferred foliation in which time is given by locally non-rotating vector field. For the case of Kerr, this is known as Boyer-Linquist time. In terms of our formalism the intrinsic geometry on such a constant time slice of Kerr can be written as

$$
\begin{aligned}
q_{i j} d x^{i} d x^{j} & =A h_{i j} d x^{i} d x^{j}+B d \phi^{2} \\
A & =\frac{r^{2}+a^{2} \cos ^{2} \theta}{R^{2}} \\
B & =\frac{r^{2}+a^{2}}{R^{2}}+\frac{2 m r a^{2} \sin ^{4} \theta}{R^{4}} \\
h_{i j} d x^{i} d x^{j} & =d R^{2}+R^{2} d \theta^{2} \\
R & :=\frac{1}{4}\left(\sqrt{r-2 m+2 \sqrt{m^{2}-a^{2}}}+\sqrt{r-2 m-2 \sqrt{m^{2}-a^{2}}}\right)^{2}
\end{aligned}
$$

where $r$ is the usual Boyer-Lindquist radial function and $R, \theta$ are polar coordinates in the half-planes of constant $\phi$. The metric $h_{i j}$ in these planes is seen to be manifestly flat.

Since we will be considering multiple black holes, it may be helpful to discuss this form for the spatial part of the Kerr metric a little further. If $A \equiv B$, the spatial metric would be conformally flat. That $A$ and $B$ differ makes the conformal flatness formalism inapplicable. It is interesting to note the limiting behavior of these functions.

$$
\begin{array}{ll}
A=1+\frac{2 m}{r}+\mathcal{O}\left(\frac{1}{r^{2}}\right) & r \rightarrow \infty \\
B \geq A & \text { everywhere } \\
\frac{B}{A}=1+\frac{a^{2} \sin ^{2} \theta}{r^{2}}+\mathcal{O}\left(\frac{1}{r^{3}}\right) & r \rightarrow \infty \\
\frac{B}{A} \rightarrow 1+\mathcal{O}\left(a^{2}\right) & a \rightarrow 0 \\
\frac{B}{A} \rightarrow 1+\mathcal{O}\left(\sin ^{2} \theta\right) & \theta \rightarrow 0
\end{array}
$$

Thus we see that $B \neq A$, but differs from $A$ minimally except in a region near each hole and off the symmetry axis. Note that this description of the Kerr data is similar to that studied by Brandt and Seidel ${ }^{6}$. They studied initial data where the Kerr spatial metric was modified by the addition of a Brill wave, which changes the ratio $B / A$.

In Boyer-Lindquist coordinates, the only non-vanishing components of the extrinsic curvature for a hypersurface of constant $t$ are $K_{r \phi}$ and $K_{\theta \phi}$. From formula 2 of last section, we know that these can be expressed in terms of a potential function $u(r, \theta)$. Doing the computation, we find that the potential responsible for these two non-vanishing components is

$$
u(r, \theta)=\frac{(1+\cos \theta)^{2}}{r^{2}+a^{2} \cos ^{2} \theta}\left(r^{2} \cos \theta-a^{2} \cos \theta-2 r^{2}\right) .
$$

We know that this potential function is the solution to a Hamilton-Jacobi equation. To find out which solution, we can inquire into the congruence of characteristic curves with which 
it is associated. Recall that these curves are orthogonal to the level curves of $u$. Having the explicit expressions at hand, we could formulate this orthogonal trajectory problem concretely and attempt to solve it. However, for our purposes such details are unnecessary, and we shall content ourselves with a qualitative discussion.

First, let us note that in the region $r>a$ and away from the axis of symmetry, $u$ is non-singular and its gradient does not vanish. As a consequence, the curves we are interested in will be smooth and free from bifurcations and fixed points. To determine their topology, we shall consider the rays $\theta=0, \pi / 2, \pi$. On the ray $\theta=\pi / 2, u$ is constant, so the characteristic curves must be orthogonal to this ray. Examining the metric $h$, we see this means that they must point along the $\theta$ direction. Near the symmetry axis, $\theta=0$, the gradient of $u$ goes to zero, so we shall make an expansion in powers of $\theta$.

$$
u(r, \theta)=-4+\frac{3 r^{2}-a^{2}}{4 r^{2}+4 a^{2}} \theta^{4}+\mathcal{O}\left(\theta^{6}\right)
$$

From this, we see that the gradient of $u$ is parallel to the symmetry axis for small $\theta$ and that its norm vanishes like $\theta^{3}$. Hence the orthogonal trajectories will point at right angles towards or away from this axis. By symmetry, the same situation will occur near the other half of the axis, $\theta=\pi$. Combining these observations, we conclude that the various curves of our congruence start out perpendicular to one end of the symmetry axis, arch around the black hole, and return perpendicular to the other end of the symmetry axis.

To generate this initial data set for Kerr metric within our formalism, we first have to specify the spatial metric by choosing $A$ and $B$ as given in equation 7 . Then, making the choices $K=K_{\phi \phi}=0$ and specifying that the congruence of curves needed for the construction of $K_{r \phi}$ and $K_{\theta \phi}$ meet the symmetry axis perpendicularly will guarantee that the correct extrinsic curvature comes out of the construction. The one remaining choice is the overall sign of the extrinsic curvature, which determines the sign of the angular momentum. This choice can be made by specifying on which side of the symmetry axis the characteristics originate.

4.Two Black Holes Given an axially symmetric spatial metric, our formalism shows how to find an extrinsic curvature which will satisfy the initial value problem of general relativity. Our strategy for representing two rotating black holes will be to choose a spatial metric which resembles the spatial metric of the Kerr solution in three regions. These are the two regions near the chosen locations for the black holes on the symmetry axis and the region near spatial infinity. Then we will use the methods described above to calculate an extrinsic curvature which satisfies the initial value problem. In general, there will be many solutions from which we will select one that is consistent with the description of the Kerr extrinsic curvature whenever the spatial metric approaches that of the Kerr solution.

The first step is to construct a three-metric which has the appropriate behavior in the near and far limits. One way to do this is to use the form given above for $A$ and $B$ of the Kerr metric and replace $\frac{R}{m}, \frac{a}{m}$, and $\sin \theta$, with functions which approach the correct values in the limits near one of the holes or away from both. Let the Kerr metrics approached near each of the two holes have parameters $\left(m_{1}, a_{1}\right)$ and $\left(m_{2}, a_{2}\right)$. Then we want the parameters of the metric approached at infinity to correspond to an appropriate sum of the masses and angular moments associated with the two holes. Thus we want

$$
\begin{gathered}
m_{\mathrm{far}}=m_{1}+m_{2} \\
\text { and } \\
a_{\mathrm{far}} m_{\mathrm{far}}=a_{1} m_{1}+a_{2} m_{2} .
\end{gathered}
$$


More explicitly then, if we rewrite the quantities $A$ and $B$ for the Kerr metric with $m_{\text {far }}$ scaled to unity and replace $R, a$, and $\sin \theta$ with $\tilde{R}, \tilde{a}$, and $\tilde{s}$, which are functions of $x$ and $y$, we have

$$
\begin{aligned}
& A=\frac{r(\tilde{R})^{2}+\tilde{a}^{2}\left(1-\tilde{s}^{2}\right)}{\tilde{R}^{2}} \\
& B=\left(\frac{r(\tilde{R})^{2}+\tilde{a}^{2}}{\tilde{R}^{2}}+\frac{2 r \tilde{R}+\tilde{a}^{2} \tilde{s}^{2}}{\tilde{R}^{4}}\right) r^{2} \sin ^{2} \theta
\end{aligned}
$$

We leave the expression " $R^{2} \sin ^{2} \theta$ " appearing in $B$ unchanged since this gives the distance from the symmetry axis and does not depend on the location of the black holes. In order for our initial data to behave properly in the three limits, we specify the following limits on the new functions:

$$
\begin{aligned}
& \left.\begin{array}{lll}
\tilde{R} & \rightarrow \frac{R_{1}}{m_{1}} \\
\tilde{s}^{2} \rightarrow \frac{R^{2} \sin ^{2} \theta}{R_{1}^{2}}
\end{array}\right\} \quad \frac{R_{1}}{m_{1}} \rightarrow 0 \\
& \tilde{a} \rightarrow \frac{a_{1}}{m_{1}} \quad \\
& \left.\begin{array}{lll}
\tilde{R} & \rightarrow \frac{R_{2}}{m_{2}} \\
\tilde{s}^{2} \rightarrow \frac{R^{2} \sin ^{2} \theta}{R_{2}^{2}}
\end{array}\right\} \quad \begin{array}{l}
\frac{R_{2}}{m_{2}} \rightarrow 0
\end{array} \\
& \tilde{a} \rightarrow \frac{a_{2}}{m_{2}} \quad \text {, } \\
& \left.\begin{array}{ll}
\tilde{R} & \rightarrow R \\
\tilde{s}^{2} & \rightarrow \sin ^{2} \theta \\
\tilde{a} & \rightarrow \frac{a_{1} m_{1}+a_{2} m_{2}}{m^{2}}
\end{array}\right\} \\
& \frac{R_{1}}{m_{1}} \text { and } \frac{R_{2}}{m_{2}} \rightarrow \infty
\end{aligned}
$$

Here $R$ and $\theta$ are polar coordinates on the conformally flat two-space and $R_{1}$ and $R_{2}$ are the distances from the chosen locations of the black holes in the flat metric. The third limit is the same as the first with 1 and 2 interchanged. A natural choice for the function $\tilde{R}\left(R_{1}, R_{2}\right)$ with suitable limiting behavior is

$$
\tilde{R}=\frac{1}{\frac{m_{1}}{R_{1}}+\frac{m_{2}}{R_{2}}}
$$

It can be seen from equation 7 that in the case of non-spinning black holes, where we expect $\tilde{a}$ to vanish identically, $B$ becomes equal to $A$ and $A$ reduces to the conformal factor associated with the familiar Brill-Lindquist initial data for non-spinning black holes.

We can satisfy the conditions for $\tilde{s}^{2}$ by choosing

$$
\tilde{s}^{2}=\frac{R^{2} \sin ^{2} \theta}{\tilde{R}^{2}}
$$

In addition to the requirements given above, there is a much less obvious condition that we must impose to assure that we can specify a $K_{i j}$ which is consistent with the Kerr extrinsic curvature. In the case where the angular momenta for the two black holes point in opposite directions, we will need to ensure the existence of a surface separating the two black holes on which the extrinsic curvature vanishes. This is essentially because $K_{i j}$ needs to change sign 
in passing from the region near one black hole to the region near the other. If the extrinsic curvature vanishes, the Hamiltonian constraint forces the scalar curvature to vanish as well.

A straightforward way to guarantee the existence of such a surface on which the curvature scalar vanishes is to require that the gradient of $\tilde{a}$ vanishes whenever $\tilde{a}$ vanishes. This follows from the following observations:

1) With our choice of $\tilde{R}$, the scalar curvature vanishes when $\tilde{a}$ vanishes identically.

2) The metric depends on $\tilde{a}$ only in the form $\tilde{a}^{2}$. The only terms surviving in the expression for the scalar curvature evaluated at a point where $\tilde{a}=0$ have the factor $q^{i j} \tilde{a}_{i} \tilde{a}_{j}$.

A function $\tilde{a}$ which meets our requirements is

$$
\tilde{a}=\tilde{R}^{6}\left(\frac{m_{1}^{2} \sqrt[3]{a_{1}}}{R_{1}^{2}}+\frac{m_{2}^{2} \sqrt[3]{a_{2}}}{R_{2}^{2}}+\frac{\sqrt[3]{a_{1} m_{1}+a_{2} m_{2}}-m_{1}^{2} \sqrt[3]{a_{1}}-m_{2}^{2} \sqrt[3]{a_{2}}}{R_{1} R_{2}}\right)^{3}
$$

These choices for $\tilde{R}, \tilde{s}$, and $\tilde{a}$ specify a metric which meets our requirements and we will take this as our spatial metric for the rest of the discussion. There are certainly other equally well-qualified and well-motivated choices of spatial geometry satisfying the conditions we have given.

Having specified a three-metric, we now want to find an extrinsic curvature which solves the constraints and resembles the extrinsic curvature for the Kerr case in the appropriate limits. Since $K_{z z}$ and the mean curvature $K$ vanish in the Kerr case, we again choose them to vanish. This means we use the trivial solution to the equations 3 and 4 . Then what remains is to find a solution of equation 5 which reduces to the $K_{i j}$ for Kerr in the limits.

To determine $K_{r \phi}$ and $K_{\theta \phi}$, specify that, as in the Kerr case, the characteristics meet the symmetry axis perpendicularly. Then the choice of sign of the tangent vector field along these curves must be made consistent with the desired sign for the angular momenta at each hole. In the case that $a_{1}$ and $a_{2}$ differ in sign, there are two regions separated by a curve on which $\tilde{a}$ vanishes. Since we have demanded that ${ }^{(3)} \mathcal{R}$ also vanish here, we can choose the signs of the vector field independently in each of these regions without introducing a discontinuity in the extrinsic curvature.

\section{Conclusions}

Here we have described a method for generating initial data on a Cauchy slice with any 3-metric that admits a surface-orthogonal Killing field. Given such a metric, our method generates all extrinsic curvature tensors which are invariant under the motion generated by the Killing field. In particular, this gives an affirmative solution to the "half-sandwich" conjecture for this class of spatial metrics and perhaps our method will find use in studying the "thin-sandwich" problem.

We have also described a set of solutions to the initial value problem which can be interpreted as two black holes arranged along a common axis of symmetry. Although we have discussed only the two black hole solutions, it should not be difficult to generalize the technique to an arbitrary number of black holes.

Our solution has the advantage over the Bowen-York solution that the geometry in the vicinity of each singularity does approach the Kerr geometry when the black holes are well separated. We suggest these data as alternatives to the Bowen-York data for exploration because they represent well-separated spinning black holes. Still, for the case of nearby black holes, our choice of initial conditions includes a certain amount of arbitrariness that can only be resolved by a more careful consideration which takes into account the precise evolution which has brought the black holes close to each other.

\section{Acknowledgments}

This work has been supported in part by the NSF grants PHY95-14240 and PHY9507688 and by the Eberly Research Funds of The Pennsylvania State University. We would also like to thank Abhay Ashtekar, Laurent Freidel and Jorge Pullin for useful suggestions. 


\section{Bibliography}

1. A. M.Abrahams et al., Gravitational Wave Extraction and Outer Boundary Conditions by Perturbative Matching preprint gr-qc/9709082

2. G. B. Cook et al., Boosted Three-Dimensional Black Hole Evolution With Singularity Excision, preprint gr-qc/9711078

3. Jeffrey Bowen and James W. York, Jr. , Time-Asymmetric initial Data for Black Holes and Black Hole Collisions Physical Review D21 (1980) 2047

4. Reinaldo J. Gleiser, Carlos O. Nicasio, Richard H. Price, and Jorge Pullin, Evolving the Bowen-York Initial Data for Spinning Black Holes, preprint gr-qc/9710096, to be published in Physical Review D

6. Steven R. Brandt and Edward Seidel, The Evolution of Distorted Rotating Black Holes III: Initial Data, Physical Review D55 (1997) 829 
CGPG-97/12-2

\title{
A New Method for Solving the Initial Value Problem with Application to Multiple Black Holes
}

\author{
John Baker and Raymond Stanley Puzio \\ Center for Gravitational Physics and Geometry, \\ Department of Physics, Pennsylvania State University, \\ University Park, PA 16802-6300, USA
}

\begin{abstract}
This work consists of two distinct parts. In the first part we present a new method for solving the initial value problem of general relativity. Given any spatial metric with a surface orthogonal Killing field and two freely specified components of the extrinsic curvature we solve for extrinsic curvature's remaining components. For the second part, after noting that initial data for the Kerr spacetime can be derived within our formalism we construct data for axisymmetric configurations of spinning black holes. Though our method is limited to axisymmetry, it offers an advantage over the Bowen-York proceedure that our data approach those for Kerr holes in the limit of large separations and in the close limit.
\end{abstract}

